\title{
Cued Memory Reactivation during Slow-Wave Sleep Promotes Explicit Knowledge of a Motor Sequence
}

\author{
James N. Cousins, ${ }^{1}$ Wael El-Deredy, ${ }^{1}$ Laura M. Parkes, ${ }^{2}$ Nora Hennies, ${ }^{1}$ and Penelope A. Lewis ${ }^{1}$ \\ ${ }^{1}$ School of Psychological Sciences, University of Manchester, Manchester M13 9PL, United Kingdom, and ${ }^{2}$ Centre for Imaging Sciences, Institute of \\ Population Health, University of Manchester, Manchester M13 9PT, United Kingdom
}

\begin{abstract}
Memories are gradually consolidated after initial encoding, and this can sometimes lead to a transition from implicit to explicit knowledge. The exact physiological processes underlying this reorganization remain unclear. Here, we used a serial reaction time task to determine whether targeted memory reactivation (TMR) of specific memory traces during slow-wave sleep promotes the emergence of explicit knowledge. Human participants learned two 12-item sequences of button presses (A and B). These differed in both cue order and in the auditory tones associated with each of the four fingers (one sequence had four higher-pitched tones). Subsequent overnight sleep was monitored, and the tones associated with one learned sequence were replayed during slow-wave sleep. After waking, participants demonstrated greater explicit knowledge $(p=0.005)$ and more improved procedural skill $(p=0.04)$ for the cued sequence relative to the uncued sequence. Furthermore, fast spindles $(13.5-15 \mathrm{~Hz})$ at task-related motor regions predicted overnight enhancement in procedural skill $(r=0.71, p=0.01)$. Auditory cues had no effect on post-sleep memory performance in a control group who received TMR before sleep. These findings suggest that TMR during sleep can alter memory representations and promote the emergence of explicit knowledge, supporting the notion that reactivation during sleep is a key mechanism in this process.
\end{abstract}

Key words: consolidation; explicit memory; learning; reactivation; replay; slow-wave sleep

\section{Introduction}

Sleep benefits many forms of memory consolidation. It aids the assimilation of memories into existing knowledge networks (Tamminen et al., 2010, 2013), facilitates inferential thinking (Ellenbogen et al., 2007), and assists in the emergence of explicit knowledge for underlying statistical regularities (Wagner et al., 2004; Fischer et al., 2006; Yordanova et al., 2008; Drosopoulos et al., 2011; Wilhelm et al., 2013).

The spontaneous reactivation of recently learned memories during sleep has been proposed as a mechanism that may underpin the reorganization of memory traces (Born et al., 2006). In rodents, neuronal firing sequences that were expressed during encoding are reinstated in subsequent periods of sleep (Wilson and McNaughton, 1994), whereas human neuroimaging studies show reactivation of learning-related

Received March 13, 2014; revised Sept. 6, 2014; accepted Sept. 14, 2014.

Author contributions: J.N.C., W.E.-D., L.M.P., and P.A.L. designed research; J.N.C. performed research; J.N.C. and N.H. analyzed data; J.N.C. and P.A.L. wrote the paper.

This work was supported by a Medical Research Council Capacity Building PhD studentship with the Biomedical Imaging Institute at the University of Manchester. We thank the University of Manchester for supporting the Manchester Sleep Laboratory.

The authors declare no competing financial interests.

This article is freely available online through the J Neurosci Author Open Choice option.

Correspondence should be addressed to James Cousins, School of Psychological Sciences, Zochonis Building, University of Manchester, Brunswick Street, Manchester M13 9PL, UK. E-mail: james.cousins@manchester.ac.uk.

DOI:10.1523/JNEUROSCI.1011-14.2014

Copyright (c) 2014 Cousins et al.

This is an Open Access article distributed under the terms of the Creative Commons Attribution License (http://creativecommons.org/licenses/by/3.0), which permits unrestricted use, distribution and reproduction in any medium provided that the original work is properly attributed. brain regions during post-encoding sleep (Maquet et al., 2000; Peigneux et al., 2003), and this predicts subsequent post-sleep performance improvement (Peigneux et al., 2004; Yotsumoto et al., 2009).

Recent studies have intentionally elicited memory reactivation by covertly presenting cues that were paired with new memories at learning during subsequent sleep. Such targeted memory reactivation (TMR) biases consolidation of both declarative ( $\mathrm{Ra}-$ sch et al., 2007; Rudoy et al., 2009; Fuentemilla et al., 2013; Cairney et al., 2014) and procedural (Antony et al., 2012; Schönauer et al., 2014) memory in humans when implemented during slowwave sleep (SWS) but not when implemented during wakefulness. TMR is thought to influence the neural replay of recently formed memories and has been shown to bias specific neuronal firing sequences in rodents (Bendor and Wilson, 2012). Together, these findings suggest that cues provide a tool for manipulating naturally occurring memory consolidation processes.

Although TMR during sleep can enhance specific memories, its influence on other transformations of memory that occur during normal sleep, such as the emergence of explicit awareness of an implicitly learned sequence (Fischer et al., 2006), remains to be explored. Here, we build on the finding that TMR influences procedural skill consolidation across sleep (Antony et al., 2012; Schönauer et al., 2014), by using a serial reaction time task (SRTT) to examine how TMR effects the overnight emergence of explicit knowledge. Participants learned two four-finger buttonpressing sequences, in which each finger was associated with a specific auditory tone, and four separate tones (higher or lower in pitch) were used for each sequence. To cue memory replay, the tones associated with one sequence were replayed to participants 
a

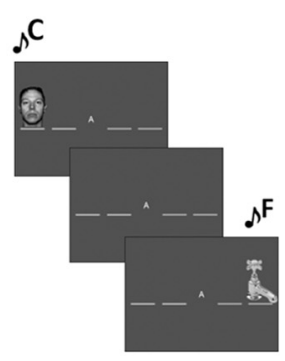

b

\begin{tabular}{c|c|c|c|}
\hline CUE & NO-CUE & CUE & NO-CUE \\
\hline 2 minutes & 2 minutes & 2 minutes & 2 minutes \\
\hline
\end{tabular}

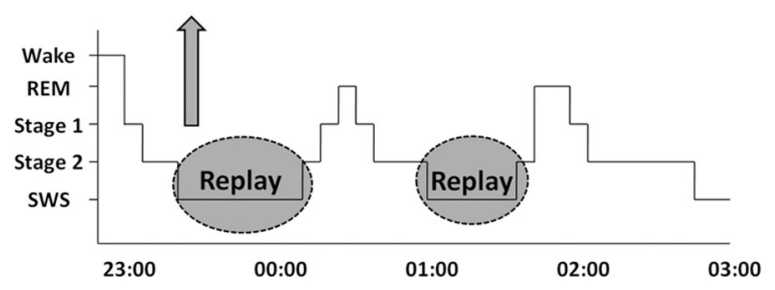

C

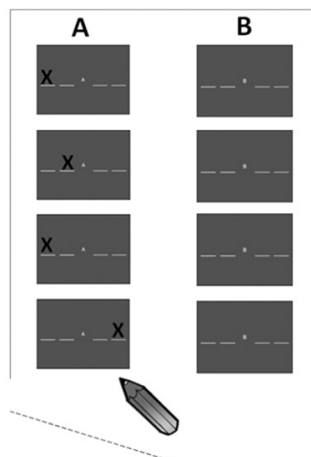

Experimental Group $(\mathrm{N}=16)$

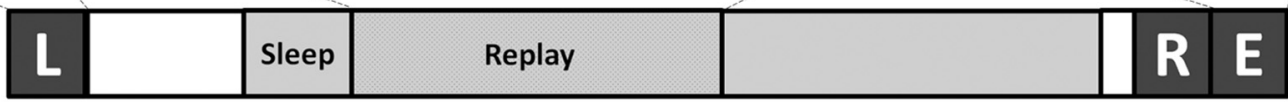

\author{
Control Group
}

$(\mathrm{N}=16)$
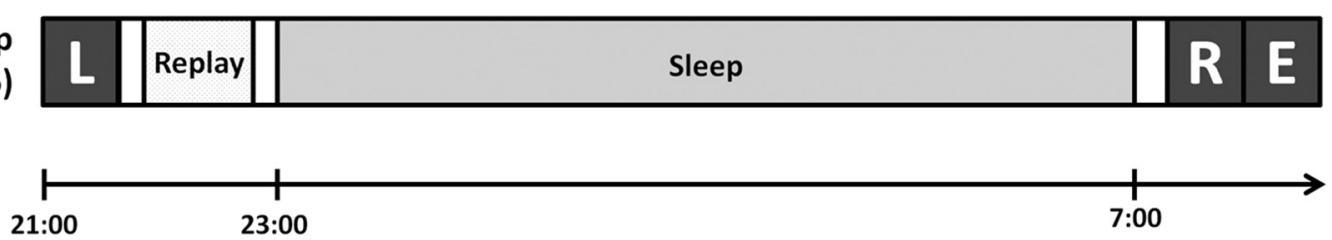

Figure 1. Experimental procedures. $\boldsymbol{a}$, SRTT learning. Visual stimulus appeared contingent with a unique tone. Correct key response was followed by a $300 \mathrm{~ms}$ interval before the next stimulus. Interleaved blocks of sequence $A$ containing low tones (4th octave: $C / D / E / F$ ) and sequence $B$ containing high tones (5th octave: $A / B / C \# / D$ ), and random blocks containing high and low tones were performed. $\boldsymbol{b}$, One sequence was played to the experimental group during SWS, with 12 repetitions (CUE) followed by silence (NO-CUE). $\boldsymbol{c}$, In the morning, participants were retested on the SRTT (R) before their explicit sequence knowledge was assessed $(\mathrm{E})$, by marking sequence order on paper.

during subsequent SWS (experimental group) or before sleep (control group). After waking the following morning, we measured explicit knowledge of sequence order and reaction times (RTs) when performing each sequence. We predicted enhanced performance on both measures for the cued sequence, indicating a role for memory replay in both forms of consolidation. The electrophysiological correlates of TMR and how they relate to consolidation are essentially unknown. We explored this by conducting a thorough analysis of spindles $(12-15 \mathrm{~Hz})$ and slow oscillations $(\sim 1 \mathrm{~Hz})$ during replay of cues and surrounding periods of SWS.

\section{Materials and Methods}

Participants. Thirty-eight healthy right-handed volunteers were screened for history of neurological, psychiatric diseases, or sleep or motor disorders and asked to abstain from caffeine and alcohol $24 \mathrm{~h}$ before testing. Participants were randomly assigned to experimental and control groups. Six participants were excluded from all analyses for corrupted sleep data $(n=1)$, RT performance at learning $>2$ SDs from group means $(n=2)$, and $>2$ SDs disparity between group mean RT for the two sequences at learning $(n=3)$. Thirty-two participants remained in experimental ( $n=16$; mean age, 24.8 years; eight females, eight males) and control ( $n=16$; mean age, 23.2 years; eight females, eight males) groups.

Design and procedure. Participants were fitted for polysomnographic recording between 7:00 P.M. and 8:00 P.M. and then performed an adapted SRTT (Nissen and Bullemer, 1987) containing interleaved blocks of two 12-item sequences: A (1-2-1-4-2-3-4-1-3-2-4-3) and B (2-4-3-2-3-1-4-2-3-1-4-1). Sequences were matched for learning difficulty, contained three repetitions of each item, and did not share strings of five or more items.

Each sequence was accompanied by either high- or low-pitched pure tones, counterbalanced across participants. Tones were musical notes grouped closely within the fourth (low; C/D/E/F) and fifth octave (high; $\mathrm{A} / \mathrm{B} / \mathrm{C} \# / \mathrm{D})$.

For each trial, a visual cue appeared with a tone in one of four spatial locations, corresponding to keys of the same configuration, and pressed with individual fingers of the left hand as quickly as possible while min- imizing errors. "A" or "B" appeared centrally on the screen indicating the sequence. Participants were not asked to explicitly learn the sequences. Visual cues were objects or faces, appearing in the same position for both sequences ( 1 , face $1 ; 2$, lamp; 3 , face $2 ; 4$, water tap). Participants were told that the nature of the cues (objects/faces) was irrelevant. Stimuli disappeared only after a correct response and were followed by a $300 \mathrm{~ms}$ intertrial interval (Fig. 1a).

Blocks containing three repetitions of a sequence were performed in pairs separated by a $2 \mathrm{~s}$ fixation cross. Each pair was followed by a $30 \mathrm{~s}$ break with RT and error-rate feedback. Blocks were interleaved pseudorandomly, with no runs of more than two blocks of the same sequence. Sequences A and B were counterbalanced across cued and uncued conditions, so that half the participants were cued with sequence A. Tones (high/low pitch) were counterbalanced across sequences. Participants performed 20 blocks each of cued (SEQ_C) and uncued sequences (SEQ_U). Four random blocks containing no repeating sequence followed, with " $R$ " displayed centrally. Half of these blocks contained tones from SEQ_C (RAND_C), and half used tones from SEQ_U (RAND_U).

The control group listened to cues while awake, $20 \mathrm{~min}$ after training. SEQ_C was played on personal computer speakers ( $48 \mathrm{~dB}$ ) imbedded in brown noise with tones $650 \mathrm{~ms}$ apart, similar to mean pre-sleep performance. Replay blocks (CUE) lasted 2 min and contained 12 sequences, followed by $2 \mathrm{~min}$ of silence (NO-CUE). To prevent rehearsal, which may influence skill and memory, participants performed a number comparison task during replay. A pair of three-digit numbers appeared on the screen, joined by a similar target number $3000 \mathrm{~ms}$ later. Participants pressed keys with the index finger of each hand to indicate which number was nearer the target. They had $3000 \mathrm{~ms}$ to respond, followed by a $500 \mathrm{~ms}$ intertrial interval.

All participants were permitted to read in bed before 11:00 P.M. lights out. Personal computer speakers played brown noise all night. In the experimental group, tones of SEQ_C were played during the first three extended periods of SWS (Fig. 1b), using the same replay protocol as in the control group. Cues were stopped immediately on arousal or leaving SWS.

Participants were awoken between 7:00 A.M. and 8:00 A.M. and allowed $20 \mathrm{~min}$ to overcome sleep inertia. At retest, 12 blocks of each sequence (SEQ_C and SEQ_U) preceded 12 repetitions of each random 
a

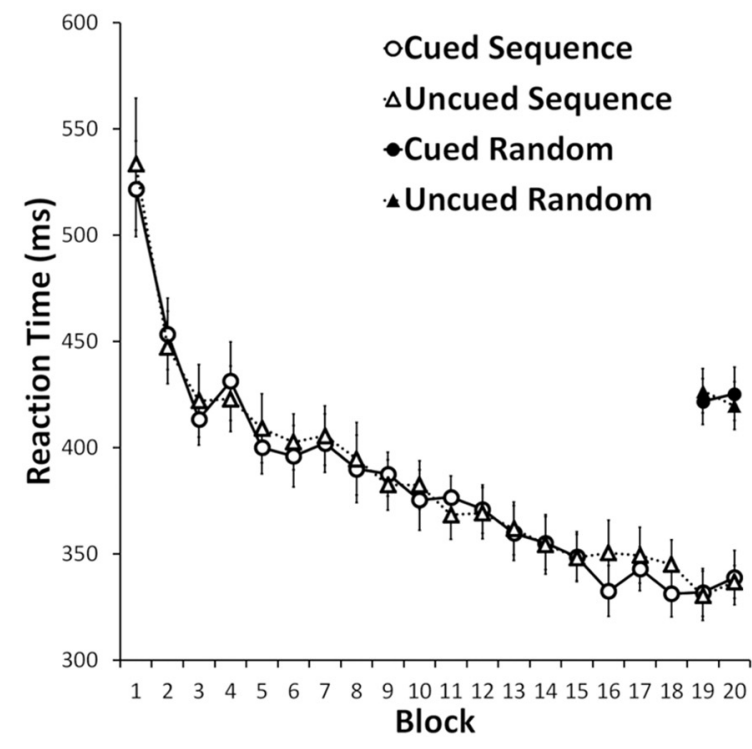

C

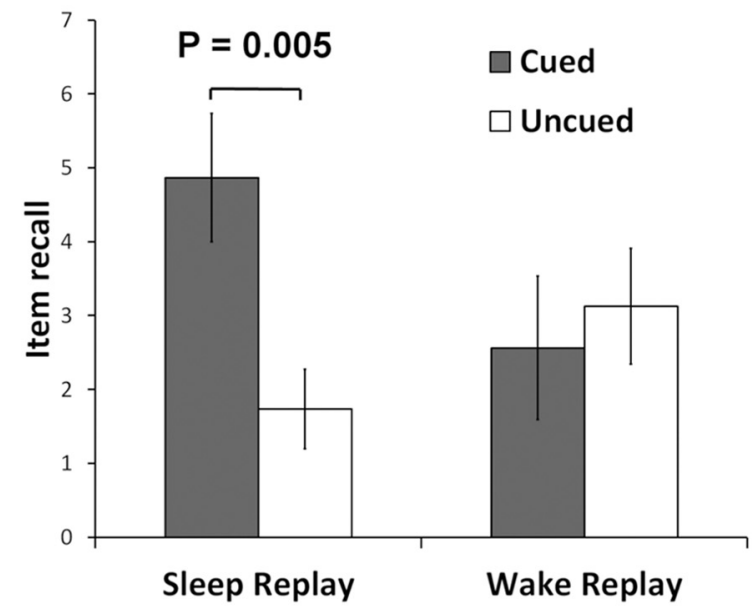

e

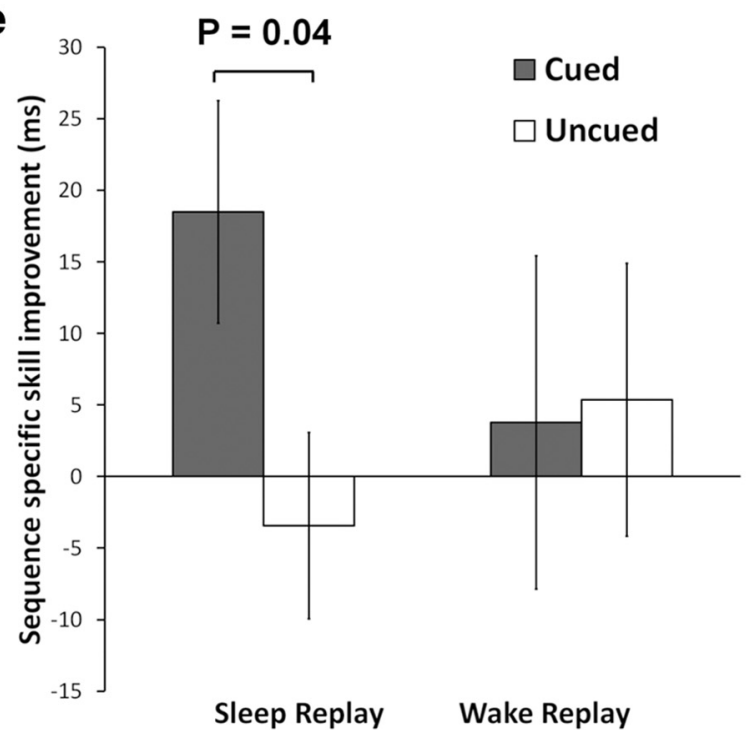

b

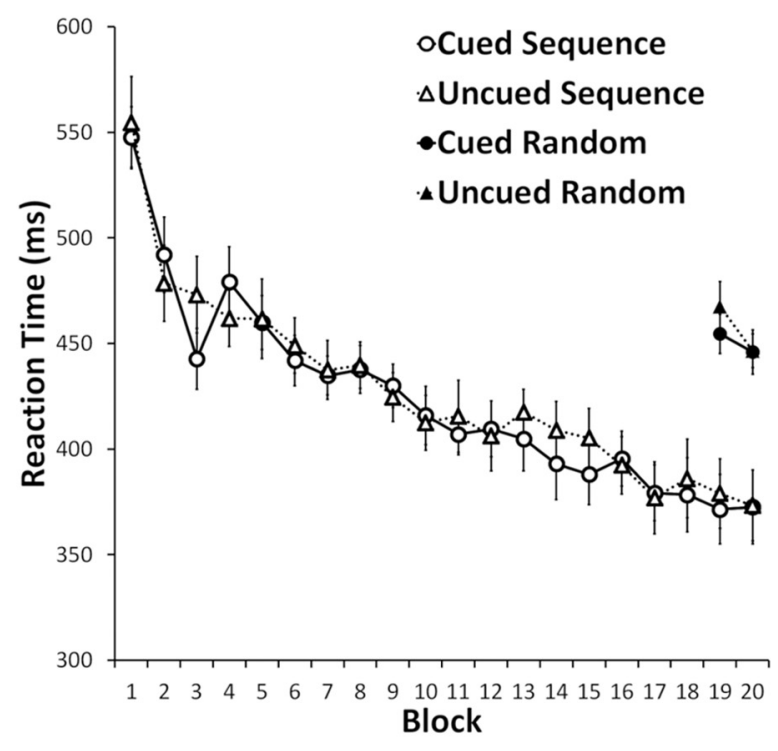

d

CUE PERIOD

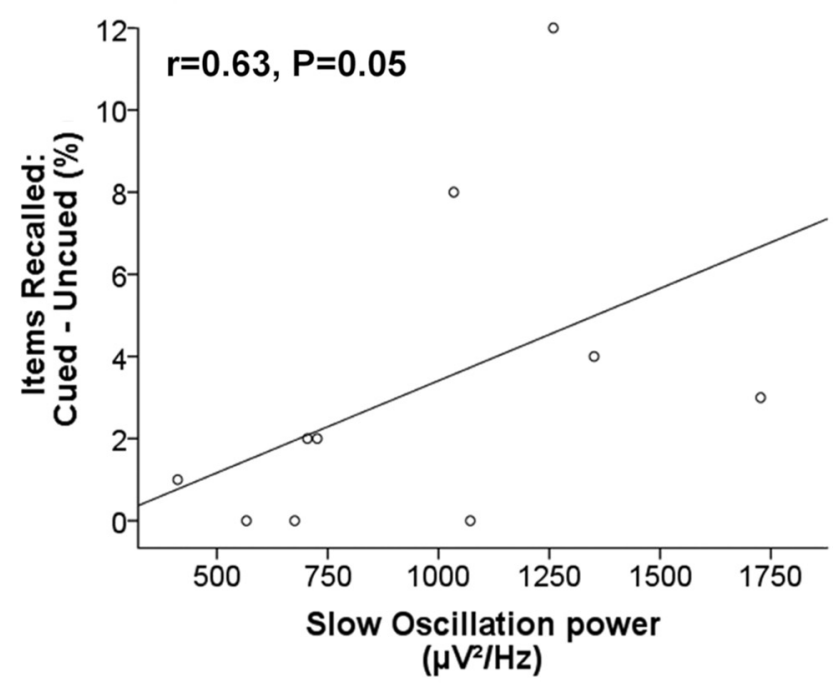

f

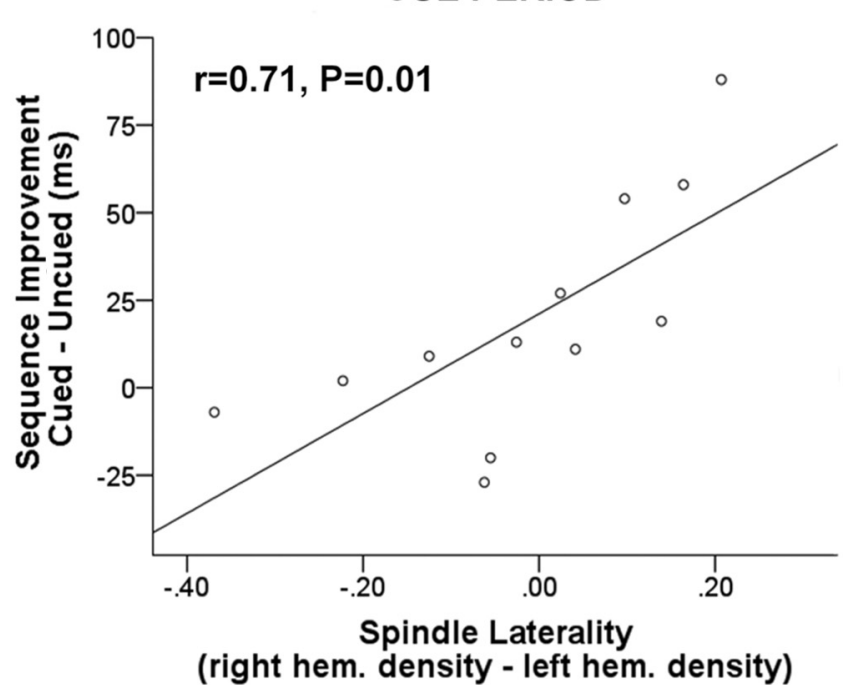


Table 1. SRTT and explicit recall data from the experimental and control groups

\begin{tabular}{cccccc}
\hline & \multicolumn{3}{l}{ Experimental group } & & Control group \\
\cline { 2 - 3 } \cline { 5 - 6 } & Cued & Uncued & & Cued & Uncued \\
\hline SRTT test blocks mean & & & & \\
RT \pm SEM (ms) & & & & \\
Learning & & & & \\
$\quad$ Sequence & $335.6 \pm 9.3$ & $341.2 \pm 9.3$ & $375.4 \pm 15.3$ & $381.7 \pm 16.1$ \\
$\quad$ Random & $423.6 \pm 11.8$ & $424.7 \pm 10.0$ & $450.3 \pm 9.0$ & $457.0 \pm 9.5$ \\
$\quad$ Difference & $88.0 \pm 9.8$ & $83.6 \pm 8.1$ & $74.9 \pm 12.0$ & $75.3 \pm 10.9$ \\
Retest & & & & \\
$\quad$ Sequence & $301.1 \pm 11.4$ & $323.8 \pm 10.9$ & $348.8 \pm 15.9$ & $353.8 \pm 17.2$ \\
Random & $407.6 \pm 10.7$ & $403.9 \pm 11.5$ & $427.5 \pm 9.3$ & $434.5 \pm 10.0$ \\
$\quad$ Difference & $106.5 \pm 11.5$ & $80.1 \pm 11.5$ & $78.7 \pm 14.8$ & $80.7 \pm 14.8$ \\
Improvement & & & & \\
$\quad$ Sequence & $34.4 \pm 6.5$ & $17.4 \pm 5.6$ & $26.6 \pm 4.7$ & $27.9 \pm 5.6$ \\
$\quad$ Random & $16.0 \pm 6.4$ & $20.8 \pm 6.2$ & & $22.8 \pm 12.0$ & $22.5 \pm 8.5$ \\
$\quad$ Sequence skill & $18.5 \pm 7.8$ & $-3.4 \pm 6.5$ & $3.8 \pm 11.7$ & $5.4 \pm 9.5$ \\
Explicit knowledge test mean & & & & \\
$\quad$ recall \pm SEM (items) & & & & \\
Recall & $4.9 \pm 0.9$ & $1.7 \pm 0.6$ & $2.6 \pm 1.0$ & $3.1 \pm 0.8$ \\
\hline
\end{tabular}

block (RAND_C and RAND_U). "REST” was displayed centrally during the $30 \mathrm{~s}$ breaks. Order of learning (i.e., whether interleaved blocks began with sequence A or B), replay, and retest was counterbalanced across participants.

Free recall was used as the measure of explicit knowledge. Participants marked sequence order on printed out screenshots arranged vertically on a page, for each sequence, with sequence order counterbalanced (Fig. 1c). All participants except one $(n=31)$ completed the explicit test. Alertness was measured using the Stanford Sleepiness Scale.

Behavioral analysis. Pre-sleep performance comprised a mean of the last four blocks of SEQ_C and SEQ_U and two blocks of RAND_C and RAND_U. Sequence was subtracted from random RT to separate learning of the sequence from sensorimotor mapping, providing a measure of "sequence-specific skill." Post-sleep performance used a mean of the last four blocks of SEQ_C and SEQ_U and the first four blocks of RAND_C and RAND_U, minimizing differences in training between SEQ and RAND because they were performed side by side. Post-sleep performance was subtracted from pre-sleep performance to determine improvement. Trials containing incorrect button presses, before the correct press, were included. Response latencies $>1000$ ms were excluded. For explicit recall, individual items were only correct if in the correct sequence position and within a segment containing more than two consecutive correct items (Willingham and Goedert-Eschmann, 1999), minimizing the influence of guessing. To determine whether explicit recall was above chance, recall of both sequences was rescored against 10 randomly generated sequences for each participant. The mean of these 10 random sequence scores was taken as "chance," and the mean of these chance scores across all participants was taken as the average number of items that would be guessed by chance.

Mixed ANOVA and paired sample $t$ tests were used for planned comparisons of cued and uncued sequence RT and recall, except when Shapiro-Wilk tests indicated a non-normal distribution, and then Friedman's ANOVA and Wilcoxon's signed-rank tests were used. Associations between behavioral measures and EEG features were tested with Pearson's correlations or Spearman's $\rho$ for non-normal distributions. All

$\leftarrow$

Figure 2. The cueing effect and neural correlates. Pre-sleep SRTT performance across all blocks of learning in the experimental group $(\boldsymbol{a})$ and the control group $(\boldsymbol{b})$. $\boldsymbol{c}$, Cues led to significantly more correctly recalled sequence items for the experimental group but not control group. $\boldsymbol{d}$, Correlation between slow oscillation power in central electrodes and the explicit cueing effect during CUE $(n=10)$ in the experimental group. e, SRTT sequence-specific skill improvement was significantly better for the cued than uncued sequence in the experimental group only. $\boldsymbol{f}$, Spindle laterality at central electrodes predicted the procedural cueing effect in the experimental group during CUE $(n=12)$ and NO-CUE. Data are presented as mean \pm SEM. Correlations are presented with some participants removed as a result of EEG artifacts. statistical tests were two tailed, with a significance level of $p<0.05$. All means are presented in text \pm SD.

EEG recording and analysis. Scalp electrodes were attached according to the 10-20 system at 14 standard locations: F3, F4, C3, C4, C5, C6, CP3, $\mathrm{CP} 4, \mathrm{CP} 5, \mathrm{CP} 6, \mathrm{P} 7, \mathrm{P} 8, \mathrm{O} 1$, and $\mathrm{O}_{2}$, referenced to the combined mean of left and right mastoid. Left and upper electromyogram, left and right electrooculagram, and a forehead ground electrode were also attached. Impedance $<5 \Omega$ was verified at each electrode, and digital sampling rate was $200 \mathrm{~Hz}$. Data were scored by two trained sleep researchers according to standard criteria (Iber et al., 2007). The second scorer was blind to CUE/NO-CUE periods. Correlations with behavioral measures focused on groups of electrodes: two frontal (F3 and F4), two parietal (P7 and $\mathrm{P} 8$ ), and eight central (C3, C4, C5, C6, CP3, CP4, CP5, and CP6) electrodes. These groupings were excluded from analysis if any electrode had to be removed because of noise.

Complete $2 \mathrm{~min}$ epochs of CUE/NO-CUE periods were extracted for every channel and concatenated separately, providing a CUE and NOCUE time series for each participant. Adjacent CUE/NO-CUE periods were rejected if they contained visually identified artifacts (e.g., movement), followed by bandpass filtering for slow $(12-13.5 \mathrm{~Hz})$ and fast (13.5-15 Hz) spindles separately, using a linear finite impulse response filter in EEGLab version 9.0, via MATLAB 2010 (MathWorks). An automated detection algorithm (Ferrarelli et al., 2007) determined the number of spindle events at each electrode. Spindle density was calculated as total spindles divided by length of the CUE/NO-CUE period time series. To explore regional spindle effects, we subtracted spindle density in left (non-learning) from right (learning) hemispheres. Outcome measures were the difference in spindle density between left and right electrodes (lateralized spindles) for the three electrode groups in the CUE/NO-CUE periods. Power spectral density was analyzed over the CUE/NO-CUE periods using Welch's method, with power averaged over each time series. This used a $4 \mathrm{~s}$ Hamming window length with $50 \%$ overlap, focusing on slow oscillations $(0.3-1 \mathrm{~Hz})$. Outcome measures were the combined mean power within the three electrode groups in the CUE/NO-CUE periods. To correct for multiple comparisons, correlations between behavioral measures and separate EEG features were false discovery rate (FDR) corrected (Benjamini and Hochberg, 1995), a method that accounts for the expected proportion of falsely rejected hypotheses. Thus, each EEG feature (e.g., fast spindles) was corrected based on a total of six comparisons, given that we measured three groups of electrodes (frontal, central, and parietal) over two periods of interest (CUE and NO-CUE).

\section{Results}

RTs were significantly faster for sequence trials compared with random trials before sleep in both the experimental and control groups ( $p<0.001)$, confirming learning of both cued and uncued sequences. Importantly, RTs for cued and uncued sequences and randomly sequenced trials did not differ before sleep ( $p>0.3$; Fig. $2 a, b$; Table 1$)$.

To examine the effects of cueing on explicit recall, we performed a mixed Friedman's ANOVA (because explicit recall was not normally distributed) with factors group (experimental/control) and replay (cued/uncued). This showed no main effect of group $\left(F_{(1,29)}=0.19, p=0.7\right)$, a marginal main effect of replay $\left(F_{(1,29)}=4.13, p=0.05\right)$, and a significant interaction $\left(F_{(1,29)}=\right.$ 5.61, $p=0.025)$. Post hoc Wilcoxon's signed-rank tests in the experimental group showed significantly better explicit recall of the cued sequence (mean, $4.9 \pm 3.5$ ) than the uncued sequence (mean, $1.7 \pm 2.2 ; p=0.005$; Fig. $2 c$ ). Cued and uncued sequence recall did not differ in the control group $(p=0.6)$, indicating that the marginal main effect of replay was driven by the experimental group. Furthermore, the cued sequence was recalled significantly better than chance in the experimental group $(p<0.001)$, whereas the uncued sequence was not $(p=0.16)$. In the control group, un cued sequence recall was above chance (mean, $3.1 \pm$ $3.1 ; p=0.01$ ), whereas cued sequence recall was not (mean, $2.6 \pm$ $3.9 ; p=0.22)$, but these did not differ significantly $(p=0.55)$. 
Table 2. Total time spent in sleep stages

\begin{tabular}{lcc}
\hline & Experimental group (minutes \pm & Control group (minutes \pm \\
& SEM) $(n=16)$ & SEM) $(n=16)$ \\
\hline Stage 1 & $26.5 \pm 3.2$ & $28.4 \pm 5.4$ \\
Stage 2 & $226.6 \pm 9.7$ & $215.0 \pm 15.9$ \\
SWS & $111.25 \pm 4.2$ & $111.1 \pm 11.4$ \\
Rapid eye movement sleep & $81.3 \pm 5.5$ & $91.1 \pm 9.0$ \\
Total sleep time & $445.7 \pm 12.1$ & $445.6 \pm 22.6$ \\
\hline
\end{tabular}

Overall, we show that TMR during sleep, but not wakefulness, increases explicit knowledge of the cued sequence.

To explore the relationship between the influence of TMR on explicit knowledge and EEG measures during (CUE) and after (NO-CUE) TMR, we subtracted explicit uncued sequence recall from cued sequence recall for each participant, creating the "explicit cueing effect." Correlations between the explicit cueing effect and slow oscillation power were marginally significant at central ( $r=0.63, p=0.05)$ electrodes (Fig. $2 d)$ and approached significance at frontal $(r=0.53, p=0.077)$ but not parietal $(r=$ $0.4, p=0.29)$ electrodes during CUE periods. NO-CUE periods showed a much weaker relationship for frontal $(r=0.31, p=$ $0.32)$, central $(r=0.42, p=0.23)$, and parietal $(r=0.32, p=$ $0.41)$ sites. There was no significant difference in mean slow oscillation power between CUE and NO-CUE periods in any electrodes $(p>0.3)$. Thus, although cues did not appear to trigger a slow oscillation power increase, the marginal central correlation suggests that slow oscillation power during cue presentation may be linked to the effect of cues on explicit recall. In contrast, neither fast nor slow lateralized spindles (non-learning minus learning hemisphere spindle density) predicted the explicit cueing effect in frontal, central, or parietal electrodes $(p>0.18)$.

To determine the effect of TMR on procedural skill, we examined overnight enhancement of sequence-specific skill (random RT minus sequence RT) using a mixed ANOVA with the factors group (experimental/control) and replay (cued/uncued). This showed no main effect of group $\left(F_{(1,30)}=0.77, p=0.8\right)$ or replay $\left(F_{(1,30)}=2.03, p=0.2\right)$ and no interaction $\left(F_{(1,30)}=2.7, p=0.1\right)$. Experimental group sequence-specific skill increased overnight (mean, $18.5 \pm 31.1$ ) for the cued sequence and decreased for the uncued sequence (mean, $-3.4 \pm 26.0$; Fig. $2 e$ ). Planned comparisons showed that this difference was significant $\left(t_{(15)}=2.32, p=\right.$ $0.04)$. Control group sequence-specific skill improvement did not differ between cued and uncued sequences $\left(t_{(15)}=-0.15\right.$, $p=0.89)$. Thus, TMR during sleep influenced consolidation of sequence-specific skill, but we can make no firm conclusion about the differential effects of TMR in sleep and wake.

To determine how the cued sequence RT advantage relates to EEG features during sleep, we first calculated a "procedural cueing effect" by subtracting uncued from cued sequence RT improvement. Lateralized fast spindles at central electrodes predicted the procedural cueing effect during both CUE $(r=$ $0.71, p=0.01$; Fig. $2 f)$ and NO-CUE $(r=0.69, p=0.01$; FDR corrected). This was not true at frontal electrodes during CUE $(r=-0.08, p=0.8)$ and NO-CUE $(r=0.01, p=0.96)$ or parietal electrodes during CUE $(r=0.55, p=0.1)$ and NO-CUE $(r=$ $0.49, p=0.2$ ). To determine whether cues increased fast spindles, we compared the CUE and NO-CUE periods for mean spindle density (rather than laterality), finding no significant differences in frontal $(p=0.6)$, central $(p=0.8)$, or parietal $(p=0.6)$ sites. Thus, cues did not trigger a net increase in fast spindles, but fast spindles over task-related areas did predict cued sequence consolidation. Analysis of slow spindles and slow oscillation power found no significant correlations with the procedural cueing effect $(p>0.2)$.

To test for a relationship between the influence of cueing on procedural and explicit measures, we correlated two explicit measures (cued sequence recall and explicit cueing effect) against four procedural performance measures: (1) cued sequence improvement (RT improvement without subtracting from random); (2) cued sequence-specific improvement (RT improvement after subtracting from random); (3) procedural cueing effect; and (4) sequence-specific cueing effect (RT improvement after subtracting from random, cued minus uncued). No significant correlations were found $(p>0.4)$.

Error rates were typically low (3.8-10.8\% trials). Comprehensive statistical tests revealed no effect of TMR on error rates $(p>$ 0.2 ); therefore, our RT findings represent a pure gain in cued sequence performance rather than a shift in speed-accuracy tradeoff.

Standard sleep scoring confirmed that the CUE/NO-CUE periods fell entirely within SWS for 12 of 16 participants. Considering all 30 s epochs, $96 \%$ of CUE periods and $97 \%$ of NO-CUE periods were in SWS, whereas the others were in Stage 2 and were excluded from additional EEG analyses. Sleep onset time, total sleep duration, and duration of all sleep stages did not differ between groups ( $p>0.3$; Table 2). Replay began $126 \pm 55 \mathrm{~min}$ after SRTT learning in the experimental group compared with 20 min after SRTT learning in the control group. The number of sequences replayed differed within the experimental (mean, $131 \pm 41.2$ ) and control (mean, $129 \pm 36.9$ ) groups but not between groups $(p=0.9)$. Combining the CUE/NO-CUE periods gave $43.17 \pm 13$ min mean replay. To establish whether sounds disrupted sleep, we compared arousal events when sounds were playing (CUE) and not playing (NO-CUE). This showed no evidence for more events during CUE: arousals ( $p=$ $0.3)$, movements $(p=0.4)$, or awakenings $(p=0.7)$. Mean occipital alpha power, which can be an indicator of arousal, did not differ between the CUE/NO-CUE periods $(p=0.7)$. No experimental group participants reported hearing tones during the night.

\section{Discussion}

This study presents the first evidence that TMR during sleep facilitates the emergence of explicit knowledge. Explicit recall of a sequence cued during sleep was significantly greater than recall of an un-cued sequence. Response speed was also influenced by TMR during sleep, with significantly more overnight RT improvement for the cued than uncued sequence. This bias was predicted by fast spindles at motor regions in the learning hemisphere.

Explicit awareness of implicitly learned SRTT sequences can emerge spontaneously after nocturnal sleep (Fischer et al., 2006). The gradual transition between implicit and explicit knowledge facilitates adaptation to a changing environment, and our data suggest that TMR can bias this otherwise spontaneous process. This result suggests that memory reactivation may underpin the emergence of explicit memory during offline consolidation and lends strong support to the active systems consolidation model (Diekelmann and Born, 2010), which proposes that memories are actively reorganized through reactivation during SWS.

The hippocampus has a central role in memory consolidation during sleep (Diekelmann and Born, 2010), and we observed that TMR influences consolidation of the hippocampal-dependent SRTT (Schendan et al., 2003), whereas non-hippocampal tasks remain to be cued successfully during sleep. Explicit recall of an 
implicitly learned sequence after sleep has been linked to postsleep enhancement in hippocampal activity (Wilhelm et al., 2013). Additional work will determine whether the behavioral changes we observed after TMR are also associated with this type of long-term plasticity.

Slow-wave activity (SWA) is strongly linked to declarative memory consolidation, and the marginally significant positive relationship we observed between the explicit cueing effect and slow oscillation power during cueing tentatively supports this association. This builds on our previous finding that SWS modulates TMR's influence upon declarative memory (Cairney et al., 2014) and predicts abstraction (Durrant et al., 2011). Importantly, Marshall et al. (2006) demonstrated that slow oscillations causally affect consolidation, whereas Wilhelm et al. (2013) found that post-training SWA predicts the emergence of explicit knowledge for an implicitly learned SRTT. However, we do advise caution when interpreting this relationship, because our correlation was at a different electrode site to that reported by Wilhelm et al. (2013) and did not survive correction for multiple comparisons.

In procedural consolidation, TMR during sleep influenced sequence-specific skill but not stimulus-response mapping. These findings are consistent with previous observations of spontaneous consolidation of SRTT sequences during sleep, in which nocturnal sleep preferentially consolidated the sequence rather than the mapping component (Robertson et al., 2004; Spencer et al., 2006), and build on this work by suggesting that TMR specifically biases procedural consolidation in favor of the cued sequence. This idea links to the finding that TMR of half an SRTT sequence enhanced performance accuracy of only that portion of the sequence (Schönauer et al., 2014). In addition, our study is the first to show that TMR influences response speed in a procedural skill rather than accuracy (Antony et al., 2012; Schönauer et al., 2014). However, note that the nonsignificant interaction means that we cannot draw conclusions about the differential effects of cueing in sleep and wake.

The predictive relationship we observed between task-specific fast spindles and enhanced RTs builds on previous findings regarding the role of spindles in procedural memory consolidation. Spindle density at task-related motor regions was shown to predict post-sleep improvement on finger tapping (Nishida and Walker, 2007), and fast spindles may be preferentially involved in consolidation, because they increase after motor learning (Barakat et al., 2011). Our correlations with fast (13.5-15 Hz), but not slow $(12-13.5 \mathrm{~Hz})$, spindles further supports a functional distinction between the two. Together, these findings support a role for regionally specific spindles in procedural consolidation, with TMR biasing consolidation in conjunction with spindles.

Interestingly, behavioral performance measures for explicit and procedural memory consolidation did not correlate, despite the similar pattern exhibited by group means for these measures. This could indicate that procedural and declarative memory systems supporting these two measures are influenced independently by TMR, resulting in differential cueing effects within each participant. However, explicit sequence knowledge is often tightly linked to RTs in the SRTT (Spencer et al., 2006), and, despite the absence of a significant relationship between our procedural and explicit measures, it remains possible that improved procedural performance after TMR facilitated explicit learning during SRTT retest. Additional work should disentangle this relationship. Antony et al. (2012) found no effect of TMR on explicit knowledge and spindle correlations slightly anterior to those we observed at central electrodes. This may stem from methodological differences between the studies, including differences in nap sleep architecture relative to overnight sleep, a larger number of TMR cues in our study, and the fact that our sound cues were contingent on visual stimuli rather than motor responses. The different spindle correlations may simply reflect issues of determining precise neural sources with a relatively small number of electrodes. Of note, the SRTT is well established as a paradigm for exploration of interactions between implicit and explicit learning (Fischer et al., 2006; Drosopoulos et al., 2011; Wilhelm et al., 2013), whereas the task used by Antony et al. (2012) has not been used for this purpose. Importantly, both studies demonstrate that there is no clear association between the effects of TMR on implicit and explicit memory.

Our behavioral data tentatively support a finite consolidation resource during sleep. Because TMR was ineffective when applied before sleep, the control group demonstrates the sequence consolidation occurring after normal unstimulated sleep. Interestingly, both procedural and explicit performance measures for the control group fell midway between the same measures for cued and uncued sequences in the experimental group (Fig. 2c,e). This pattern was also observed by Antony et al., who speculated that it implies TMR produces a consolidation bias rather than a pure gain. Similarly, if spindles and slow oscillations reflect the electrophysiological correlates of reactivation, then the inability of cues to increase them also suggests a finite consolidation resource.

Bendor and Wilson (2012) observed that sound cues did not increase the amount of reactivation of neuronal ensembles in the hippocampi of rodents but could nevertheless bias the content of subsequent replay events up to $10 \mathrm{~s}$ after cueing. Similarly, our comparison of CUE and NO-CUE periods found no evidence that cues increase slow oscillation power or fast spindles, which are considered to be neural correlates of sleep-dependent memory consolidation (Diekelmann and Born, 2010). We observed correlations between lateralized spindles and procedural memory consolidation both during replay (CUE) and also during 2 min periods of silence after replay (NO-CUE). This could indicate trait-like individual differences, whereby people with naturally right-lateralized spindles benefit more from TMR of our right-lateralized task. Additionally, cues in our study may have triggered a short-lived increase in SWA (Rihm et al., 2014) or a continued increase throughout NO-CUE periods and subsequent SWS (Cairney et al., 2014). Our cueing procedure was not designed to discern between these different accounts, so additional work is needed to explore these possibilities.

In relation to the cueing procedure itself, a remaining question regards whether TMR cues must exactly match the cues associated with learning for TMR to influence consolidation. In our study, TMR cues did not follow the exact temporal rhythm experienced during learning, because SRTT performance includes an inconsistent gap between stimuli that depends on response speed, while tones were spaced evenly during replay. The success of this procedure shows that cues do not need precisely the same timing as the learning experience to reactivate memories.

A limitation to our study is that, to avoid interference with SRTT performance, explicit knowledge was not tested before sleep. Therefore, it remains possible that TMR during sleep may not have promoted the emergence of explicit knowledge but instead protected existing explicit knowledge against decay. Additionally, the difference in delay of TMR between groups could potentially account for why cueing was unsuccessful in the control group, because initial consolidation processes may need to be completed before TMR is effective. Notably, in the study by Antony et al. 
(2012), TMR during wake did not influence consolidation even when this delay was matched across sleep and wake groups.

This study did not examine other sleep stages, so the specificity of reactivation to SWS remains unclear. Reactivation of brain regions involved in SRTT learning has been identified in rapid eye movement (Maquet et al., 2000; Peigneux et al., 2003), whereas others propose that lighter sleep stages may be important (Genzel et al., 2014). Additional work is needed to determine the role of these stages in reactivation.

The gradual emergence of explicit awareness for statistical regularities forms a critical part of learning and directing appropriate behavior and reflects a form of reorganization of memory traces in the brain. This occurs preferentially during sleep and provides evidence for an active consolidation process. We show that experimentally manipulating reactivation of a procedural memory biases explicit knowledge, suggesting a causal role for reactivation during sleep in this type of consolidation. The complex neuronal processes of reactivation remain to be discovered, but our data suggest distinct roles for slow oscillations and fast spindles for explicit knowledge and procedural memory, respectively.

\section{Notes}

Supplemental material for this article is available at http://personalpages. manchester.ac.uk/staff/plewis/Cousins_et_al_2014.pdf. Tables of error performance and correlations between behavioral measures and EEG features, as well as a movie of the serial reaction time task. This material has not been peer reviewed.

\section{References}

Antony JW, Gobel EW, O'Hare JK, Reber PJ, Paller KA (2012) Cued memory reactivation during sleep influences skill learning. Nat Neurosci 15: 1114-1116. CrossRef Medline

Barakat M, Doyon J, Debas K, Vandewalle G, Morin A, Poirier G, Martin N, Lafortune M, Karni A, Ungerleider LG, Benali H, Carrier J (2011) Fast and slow spindle involvement in the consolidation of a new motor sequence. Behav Brain Res 217:117-121. CrossRef Medline

Bendor D, Wilson MA (2012) Biasing the content of hippocampal replay during sleep. Nat Neurosci 15:1439-1444. CrossRef Medline

Benjamini Y, Hochberg Y (1995) Controlling the false discovery rate: a practical and powerful approach to multiple testing. J R Stat Soc Ser B Stat Methodol 57:289-300.

Born J, Rasch B, Gais S (2006) Sleep to remember. Neuroscientist 12:410424. CrossRef Medline

Cairney SA, Durrant SJ, Hulleman J, Lewis PA (2014) Targeted memory reactivation during slow wave sleep facilitates emotional memory consolidation. Sleep 37:701-707, 707A. CrossRef Medline

Diekelmann S, Born J (2010) The memory function of sleep. Nat Rev Neurosci 11:114-126. CrossRef Medline

Drosopoulos S, Harrer D, Born J (2011) Sleep and awareness about presence of regularity speed the transition from implicit to explicit knowledge. Biol Psychol 86:168-173. CrossRef Medline

Durrant SJ, Taylor C, Cairney S, Lewis PA (2011) Sleep-dependent consolidation of statistical learning. Neuropsychologia 49:1322-1331. CrossRef Medline

Ellenbogen JM, Hu PT, Payne JD, Titone D, Walker MP (2007) Human relational memory requires time and sleep. Proc Natl Acad Sci U S A 104:7723-7728. CrossRef Medline

Ferrarelli F, Huber R, Peterson MJ, Massimini M, Murphy M, Riedner BA, Watson A, Bria P, Tononi G (2007) Reduced sleep spindle activity in schizophrenia patients. Am J Psychiatry 164:483-492. CrossRef Medline

Fischer S, Drosopoulos S, Tsen J, Born J (2006) Implicit learning-explicit knowing: a role for sleep in memory system interaction. J Cogn Neurosci 18:311-319. CrossRef Medline

Fuentemilla L, Miró J, Ripollés P, Vilà-Balló A, Juncadella M, Castañer S, Salord N, Monasterio C, Falip M, Rodríguez-Fornells A (2013) Hippocampus-dependent strengthening of targeted memories via reacti- vation during sleep in humans. Curr Biol 23:1769-1775. CrossRef Medline

Genzel L, Kroes MC, Dresler M, Battaglia FP (2014) Light sleep versus slow wave sleep in memory consolidation: a question of global versus local processes? Trends Neurosci 37:10-19. CrossRef Medline

Iber C, Ancoli-Israel S, Chesson A, Quan SF (2007) The AASM manual for the scoring of sleep and associated events: rules, terminology and technical specifications. Westchester, IL: American Academy of Sleep Medicine.

Maquet P, Laureys S, Peigneux P, Fuchs S, Petiau C, Phillips C, Aerts J, Del Fiore G, Degueldre C, Meulemans T, Luxen A, Franck G, Van Der Linden M, Smith C, Cleeremans A (2000) Experience-dependent changes in cerebral activation during human REM sleep. Nat Neurosci 3:831-836. CrossRef Medline

Marshall L, Helgadóttir H, Mölle M, Born J (2006) Boosting slow oscillations during sleep potentiates memory. Nature 444:610-613. CrossRef Medline

Nishida M, Walker MP (2007) Daytime naps, motor memory consolidation and regionally specific sleep spindles. PLoS One 2:e341. CrossRef Medline

Nissen MJ, Bullemer P (1987) Attentional requirements of learning-evidence from performance-measures. Cognit Psychol 19:1-32. CrossRef

Peigneux P, Laureys S, Fuchs S, Destrebecqz A, Collette F, Delbeuck X, Phillips C, Aerts J, Del Fiore G, Degueldre C, Luxen A, Cleeremans A, Maquet P (2003) Learned material content and acquisition level modulate cerebral reactivation during posttraining rapid-eye-movements sleep. Neuroimage 20:125-134. Medline

Peigneux P, Laureys S, Fuchs S, Collette F, Perrin F, Reggers J, Phillips C, Degueldre C, Del Fiore G, Aerts J, Luxen A, Maquet P (2004) Are spatial memories strengthened in the human hippocampus during slow wave sleep? Neuron 44:535-545. CrossRef Medline

Rasch B, Büchel C, Gais S, Born J (2007) Odor cues during slow-wave sleep prompt declarative memory consolidation. Science 315:1426-1429. CrossRef Medline

Rihm JS, Diekelmann S, Born J, Rasch B (2014) Reactivating memories during sleep by odors: odor specificity and associated changes in sleep oscillations. J Cogn Neurosci 26:1806-1818. CrossRef Medline

Robertson EM, Pascual-Leone A, Press DZ (2004) Awareness modifies the skill-learning benefits of sleep. Curr Biol 14:208-212. CrossRef Medline

Rudoy JD, Voss JL, Westerberg CE, Paller KA (2009) Strengthening individual memories by reactivating them during sleep. Science 326:1079-1079. CrossRef Medline

Schendan HE, Searl MM, Melrose RJ, Stern CE (2003) An fMRI study of the role of the medial temporal lobe in implicit and explicit sequence learning. Neuron 37:1013-1025. CrossRef Medline

Schönauer M, Geisler T, Gais S (2014) Strengthening procedural memories by reactivation in sleep. J Cogn Neurosci 26:143-153. CrossRef Medline

Spencer RMC, Sunm M, Ivry RB (2006) Sleep-dependent consolidation of contextual learning. Curr Biol 16:1001-1005. CrossRef Medline

Tamminen J, Payne JD, Stickgold R, Wamsley EJ, Gaskell MG (2010) Sleep spindle activity is associated with the integration of new memories and existing knowledge. J Neurosci 30:14356-14360. CrossRef Medline

Tamminen J, Lambon Ralph MA, Lewis PA (2013) The role of sleep spindles and slow-wave activity in integrating new information in semantic memory. J Neurosci 33:15376-15381. CrossRef Medline

Wagner U, Gais S, Haider H, Verleger R, Born J (2004) Sleep inspires insight. Nature 427:352-355. CrossRef Medline

Wilhelm I, Rose M, Imhof KI, Rasch B, Büchel C, Born J (2013) The sleeping child outplays the adult's capacity to convert implicit into explicit knowledge. Nat Neurosci 16:391-393. CrossRef Medline

Willingham DB, Goedert-Eschmann K (1999) The relation between implicit and explicit learning: evidence for parallel development. Psych Sci 10:531. CrossRef

Wilson MA, McNaughton BL (1994) Reactivation of hippocampal ensemble memories during sleep. Science 265:676-679. CrossRef Medline

Yordanova J, Kolev V, Verleger R, Bataghva Z, Born J, Wagner U (2008) Shifting from implicit to explicit knowledge: different roles of early- and late-night sleep. Learn Mem 15:508-515. CrossRef Medline

Yotsumoto Y, Sasaki Y, Chan P, Vasios CE, Bonmassar G, Ito N, Náñez JE, Shimojo S, Watanabe T (2009) Location-specific cortical activation changes during sleep after training for perceptual learning. Curr Biol 19:1278-1282. CrossRef Medline 